\title{
Global attractors for the plate equation with nonlocal nonlinearity in unbounded domains
}

\author{
Zehra Arat, Azer Khanmamedov, and Sema Simsek
}

Communicated by Sergey Zelik, received August 8, 2014.

\begin{abstract}
We consider the initial value problem for the semilinear plate equation with nonlocal nonlinearity. We prove the existence of global attractor and then establish the regularity and finite dimensionality of this attractor.
\end{abstract}

\section{Contents}

1. Introduction

2. Existence of the global attractor

3. Regularity of the global attractor

4. Finite dimensionality of the global attractor

\section{Introduction}

The main aim of this paper is to study the long time dynamics (in terms of attractors) of the plate equation

$$
\begin{gathered}
u_{t t}+\Delta^{2} u+\alpha(x) u_{t}+\lambda u \\
-f\left(\|\nabla u(t)\|_{L^{2}\left(\mathbb{R}^{n}\right)}\right) \Delta u=h(x), \quad(t, x) \in(0, \infty) \times \mathbb{R}^{n},
\end{gathered}
$$

with initial data

$$
u(0, x)=u_{0}(x), \quad u_{t}(0, x)=u_{1}(x), \quad x \in \mathbb{R}^{n},
$$

where $\lambda>0, h \in L^{2}\left(\mathbb{R}^{n}\right)$ and the functions $\alpha(\cdot), f(\cdot)$ satisfy the following conditions:

$$
\alpha \in L^{\infty}\left(\mathbb{R}^{n}\right), \quad \alpha(\cdot) \geq \alpha_{0}>0 \text { a.e. in } \mathbb{R}^{n}
$$

2000 Mathematics Subject Classification. 35B41, 35G20, 37L30, 74K20.

Key words and phrases. Plate equation, global attractor. 


$$
f \in C^{1}\left(\mathbb{R}^{+}\right), \quad f(z) \geq 0, \text { for all } z \in \mathbb{R}^{+} .
$$

Denoting $A\left(w_{1}, w_{2}\right)=\left(w_{2},-\Delta^{2} w_{1}-\lambda w_{1}-\alpha(\cdot) w_{2}\right), D(A)=H^{4}\left(\mathbb{R}^{n}\right) \times H^{2}\left(\mathbb{R}^{n}\right)$ and $\Phi\left(w_{1}, w_{2}\right)=\left(0, f\left(\left\|\nabla w_{1}\right\|_{L^{2}\left(\mathbb{R}^{n}\right)}\right) \Delta w_{1}+h\right)$, we can reduce (1.1)-(1.2) to the problem

$$
\left\{\begin{array}{l}
\frac{d}{d t}\left(u, u_{t}\right)=A\left(u, u_{t}\right)+\Phi\left(u, u_{t}\right) \\
\left(u(0), u_{t}(0)\right)=\left(u_{0}, u_{1}\right)
\end{array}\right.
$$

in $H^{2}\left(\mathbb{R}^{n}\right) \times L^{2}\left(\mathbb{R}^{n}\right)$. Defining suitable equivalent norm in $H^{2}\left(\mathbb{R}^{n}\right) \times L^{2}\left(\mathbb{R}^{n}\right)$, it is easy to verify that $A$ is maximal dissipative operator in $H^{2}\left(\mathbb{R}^{n}\right) \times L^{2}\left(\mathbb{R}^{n}\right)$ and consequently it generates a linear continuous semigroup $\left\{e^{t A}\right\}_{t \geq 0}$ in $H^{4}\left(\mathbb{R}^{n}\right) \times H^{2}\left(\mathbb{R}^{n}\right)$ and $H^{2}\left(\mathbb{R}^{n}\right) \times L^{2}\left(\mathbb{R}^{n}\right)$. Since, by (1.4), the nonlinear operator $\Phi: H^{2}\left(\mathbb{R}^{n}\right) \times$ $L^{2}\left(\mathbb{R}^{n}\right) \rightarrow H^{2}\left(\mathbb{R}^{n}\right) \times L^{2}\left(\mathbb{R}^{n}\right)$ is Lipschitz continuous on bounded subsets of $H^{2}\left(\mathbb{R}^{n}\right) \times$ $L^{2}\left(\mathbb{R}^{n}\right)$, by the semigroup theory (see, for example [1, p. 56-58]), for every $\left(u_{0}, u_{1}\right) \in$ $H^{2}\left(\mathbb{R}^{n}\right) \times L^{2}\left(\mathbb{R}^{n}\right)$, the problem (1.5) has a unique local weak solution $\left(u, u_{t}\right) \in$ $C\left(\left[0, T_{\max }\right) ; H^{2}\left(\mathbb{R}^{n}\right) \times L^{2}\left(\mathbb{R}^{n}\right)\right)$. Moreover, if $\left(u_{0}, u_{1}\right) \in H^{4}\left(\mathbb{R}^{n}\right) \times H^{2}\left(\mathbb{R}^{n}\right)$, then $\left(u, u_{t}\right)$ is a strong solution of (1.5), and consequently of (1.1)-(1.2), from the class $C\left(\left[0, T_{\max }\right) ; H^{4}\left(\mathbb{R}^{n}\right) \times H^{2}\left(\mathbb{R}^{n}\right)\right)$.

Let $u \in C\left(\left[0, T_{\max }\right) ; H^{4}\left(\mathbb{R}^{n}\right)\right) \cap C^{1}\left(\left[0, T_{\max }\right) ; H^{2}\left(\mathbb{R}^{n}\right)\right)$ be local strong solution of (1.1)-(1.2). Multiplying (1.1) by $u_{t}$ and integrating over $(s, t) \times \mathbb{R}^{n}$, we get

$$
\begin{gathered}
E(u(t))+\frac{1}{2} F\left(\|\nabla u(t)\|_{L^{2}\left(\mathbb{R}^{n}\right)}^{2}\right) \\
-\int_{\mathbb{R}^{n}} h(x) u(t, x) d x+\int_{s} \int_{\mathbb{R}^{n}} \alpha(x)\left|u_{t}(\tau, x)\right|^{2} d x d \tau \\
=E(u(s))+\frac{1}{2} F\left(\|\nabla u(s)\|_{L^{2}\left(\mathbb{R}^{n}\right)}^{2}\right) \\
-\int_{\mathbb{R}^{n}} h(x) u(s, x) d x, \quad t \geq s \geq 0,
\end{gathered}
$$

where $F(z)=\int_{0}^{z} f(\sqrt{s}) d s$ for all $z \in \mathbb{R}^{+}$and

$$
E(u(t))=\frac{1}{2} \int_{\mathbb{R}^{n}}\left(\left|u_{t}(t, x)\right|^{2}+|\Delta u(t, x)|^{2}+\lambda|u(t, x)|^{2}\right) d x .
$$

Taking into account (1.3) and (1.4) in (1.6), we obtain

$$
\begin{gathered}
\left\|\left(u(t), u_{t}(t)\right)\right\|_{H^{2}\left(\mathbb{R}^{n}\right) \times L^{2}\left(\mathbb{R}^{n}\right)} \\
\leq c\left(\left\|\left(u_{0}, u_{1}\right)\right\|_{H^{2}\left(\mathbb{R}^{n}\right) \times L^{2}\left(\mathbb{R}^{n}\right)}\right), \quad t \geq 0,
\end{gathered}
$$

where $c: \mathbb{R}^{+} \rightarrow \mathbb{R}^{+}$is a nondecreasing function. The last inequality yields that the local solution $u$ can be extended to $[0, \infty)$.

Now, let $v^{(i)} \in C\left([0, \infty) ; H^{4}\left(\mathbb{R}^{n}\right)\right) \cap C^{1}\left([0, \infty) ; H^{2}\left(\mathbb{R}^{n}\right)\right)$ be strong solutions of (1.1)-(1.2) with initial data $\left(v_{0}^{(i)}, v_{1}^{(i)}\right) \in H^{4}\left(\mathbb{R}^{n}\right) \times H^{2}\left(\mathbb{R}^{n}\right), i=1$, 2. Putting $v^{(1)}$ 
and $v^{(2)}$ instead of $u$ in (1.1), subtracting the equations, multiplying the obtained equation by $\left(v_{t}-w_{t}\right)$ and integrating over $(0, t) \times \mathbb{R}^{n}$, we find

$$
\begin{gathered}
\left\|v^{(1)}(t)-v^{(2)}(t)\right\|_{H^{2}\left(\mathbb{R}^{n}\right)}+\left\|v_{t}^{(1)}(t)-v_{t}^{(2)}(t)\right\|_{L^{2}\left(\mathbb{R}^{n}\right)} \\
\leq \widetilde{c}(T, \widetilde{r})\left(\left\|v_{0}^{(1)}-v_{0}^{(2)}\right\|_{H^{2}\left(\mathbb{R}^{n}\right)}+\left\|v_{1}^{(1)}-v_{1}^{(2)}\right\|_{L^{2}\left(\mathbb{R}^{n}\right)}\right), \quad \forall t \in[0, T],
\end{gathered}
$$

where $\widetilde{c}: R_{+} \times R_{+} \rightarrow R_{+}$is a nondecreasing function with respect to each variable and $\widetilde{r}=\max \left\{\left\|\left(v_{0}^{(1)}, v_{1}^{(1)}\right)\right\|_{H^{2}\left(\mathbb{R}^{n}\right) \times L^{2}\left(\mathbb{R}^{n}\right)},\left\|\left(v_{0}^{(2)}, v_{1}^{(2)}\right)\right\|_{H^{2}\left(\mathbb{R}^{n}\right) \times L^{2}\left(\mathbb{R}^{n}\right)}\right\}$. The last inequality, together with (1.7), implies that the problem (1.1)-(1.2) generates a bounded strongly continuous semigroup $\{S(t)\}_{t \geq 0}$ in $H^{2}\left(\mathbb{R}^{n}\right) \times L^{2}\left(\mathbb{R}^{n}\right)$ by the formula $\left(u(t), u_{t}(t)\right)=S(t)\left(u_{0}, u_{1}\right)$.

The problem of investigating the asymptotic behavior of evolution equations modeling many physical phenomena has been attracting more attention over the last few decades. It is well known that the asymptotic behavior of these equations can be described by means of the attractors. The attractors for plate equations has been one of the intensively studied topics in recent years. We refer to [2-11] for attractors of plate equations with local and nonlocal nonlinearities in bounded domains. In the case of unbounded domains, there are obstacles in applying the methods given for bounded domains due to the lack of Sobolev compact embedding theorems. To overcome these obstacles one needs uniform tail estimates. One of the ways to obtain the tail estimate is to use weight functions as it was done in [12] for the reaction diffusion equation. However, the specificity of the plate equation does not allow to apply the method introduced in [12]. So as to handle the obstacles caused by the lack of Sobolev compact embedding theorem, the authors of [13-16] established the tail estimates in the time average form for the plate equations with local nonlinearities and then used the energy method.

The situation becomes more difficult when the domain is unbounded and the equation includes nonlocal nonlinearity, for example $f\left(\|\nabla u(t)\|_{L^{2}\left(\mathbb{R}^{n}\right)}\right) \Delta u(t)$ as in the case of equation (1.1). When $f(s)=s^{2}$, this nonlocal term becomes famous Berger nonlinearity (see [17]). In the unbounded domain case, the operator $\mathcal{F}(u):=$ $f\left(\|\nabla u\|_{L^{2}\left(\mathbb{R}^{n}\right)}\right) \Delta u$ which is determined by the nonlocal term mentioned above, besides being not compact, is not also weakly continuous from $H^{2}\left(\mathbb{R}^{n}\right)$ to $L^{2}\left(\mathbb{R}^{n}\right)$. So, in order to establish the asymptotic compactness which is necessary for the existence of the global attractor, we are not able to apply either the standard splitting method or the energy method devised in [18]. To overcome this difficulty, we apply compensated compactness method introduced in [19] and prove the asymptotic compactness (see Lemma 2.2) which, together with the presence of the strict Lyapunov function, leads to the existence of a global attractor. Then, by using the invariance of the global attractor and the structural property of the set of stationary points, we establish the regularity (see Theorem 3.1) and consequently, the finite dimensionality (see Theorem 4.1) of the global attractor.

Our main result is as follows:

THEOREM 1.1. Under conditions (1.3) and (1.4) the semigroup $\{S(t)\}_{t>0}$ generated by the problem (1.1)-(1.2) possesses a global attractor $\mathcal{A}$ in $H^{2}\left(\mathbb{R}^{n}\right) \times L^{2}\left(\mathbb{R}^{n}\right)$ and $\mathcal{A}=\mathcal{M}^{u}(\mathcal{N})$. Here $\mathcal{M}^{u}(\mathcal{N})$ is unstable manifold emanating from the set of stationary points $\mathcal{N}$ (for definition, see [20, p. 359]). Moreover, the global attractor 
$\mathcal{A}$ is bounded in $H^{4}\left(\mathbb{R}^{n}\right) \times H^{2}\left(\mathbb{R}^{n}\right)$ and it has finite fractal dimension.

REMARK 1.1. We note that by using the method of this paper, one can prove the existence, regularity and finite dimensionality of the global attractor for the initial boundary value problem

$$
\begin{cases}u_{t t}+\Delta^{2} u+\alpha(x) u_{t}+\lambda u & \\ -f\left(\|\nabla u(t)\|_{L^{2}(\Omega)}\right) \Delta u=h(x), & (t, x) \in(0, \infty) \times \Omega, \\ u(t, x)=\frac{\partial}{\partial \nu} u(t, x)=0, & (t, x) \in(0, \infty) \times \partial \Omega, \\ u(0, x)=u_{0}(x), \quad u_{t}(0, x)=u_{1}(x), & x \in \Omega,\end{cases}
$$

where $\Omega \subset \mathbb{R}^{n}$ is an unbounded domain with smooth boundary, $\nu$ is outer unit normal vector, $\lambda>0, h \in L^{2}(\Omega)$, the function $f(\cdot)$ satisfies the condition (1.4) and the damping coefficient $\alpha(\cdot)$ satisfies the following conditions

$$
\alpha \in L^{\infty}(\Omega), \quad \alpha(\cdot) \geq \alpha_{0}>0, \text { a.e. in } \Omega .
$$

REMARK 1.2. We also note that we critically use the strict positivity of $\alpha(\cdot)$ (see (1.3)) in the proof of asymptotic compactness of the semigroup $\{S(t)\}_{t \geq 0}$ (see Lemma 2.2). In the case when the function $\alpha(\cdot)$ is not strictly positive, for example if $\alpha(\cdot)$ vanishes in a set of positive measure, our method is not applicable. Another obstacle in this case is related to unique continuation of solutions which is important for the construction of a strict Lyapunov function. To the best of our knowledge, the unique continuation of solutions for the equations (1.1) and $(1.8)_{1}-(1.8)_{2}$, especially in the case when the force term $h(\cdot)$ vanishing in the damping region, is also an open question (see also [21]). Thus, in the case when $\alpha(\cdot)$ vanishes in a set of positive measure, the questions about long time dynamics of (1.1)-(1.2) and (1.8), in terms of attractors, are completely open.

\section{Existence of the global attractor}

In this section, we will show the existence of the global attractor. To this end, we first prove the following lemma.

Lemma 2.1. Let the conditions (1.3) and (1.4) hold. Also, assume that the sequence $\left\{v_{m}\right\}_{m=1}^{\infty}$ is bounded in $L^{\infty}\left(0, T ; H^{2}\left(\mathbb{R}^{n}\right)\right) \cap W^{1, \infty}\left(0, T ; L^{2}\left(\mathbb{R}^{n}\right)\right)$ and the sequence $\left\{\left\|\nabla v_{m}(t)\right\|_{L^{2}\left(\mathbb{R}^{n}\right)}\right\}_{m=1}^{\infty}$ is convergent, for all $t \in[0, T]$. Then, for all $\gamma>0$, there exists some $c_{\gamma}>0$ such that

$$
\begin{gathered}
\int_{0}^{t} \int_{\mathbb{R}^{n}} \tau\left(f\left(\left\|\nabla v_{m}(\tau)\right\|_{L^{2}\left(\mathbb{R}^{n}\right)}\right) \Delta v_{m}(\tau, x)-f\left(\left\|\nabla v_{l}(\tau)\right\|_{L^{2}\left(\mathbb{R}^{n}\right)}\right) \Delta v_{l}(\tau, x)\right) \times \\
\quad\left(v_{m t}(\tau, x)-v_{l t}(\tau, x)\right) d x d \tau \\
\leq \gamma \int_{0}^{t} \tau E\left(v_{m}(\tau)-v_{l}(\tau)\right) d \tau+c_{\gamma} \int_{0}^{t} E\left(v_{m}(\tau)-v_{l}(\tau)\right) d \tau \\
\quad+c_{\gamma} \int_{0}^{t} \tau E\left(v_{m}(\tau)-v_{l}(\tau)\right)\left\|v_{m t}(\tau)\right\|_{L^{2}\left(\mathbb{R}^{n}\right)}^{2} d \tau+K^{m, l}(t),
\end{gathered}
$$

for all $t \in[0, T]$, where $K^{m, l} \in C[0, T]$ and $\underset{m \rightarrow \infty}{\limsup } \limsup _{l \rightarrow \infty}\left\|K^{m, l}\right\|_{C[0, T]}=0$. 
Proof. Firstly, we have

$$
\begin{gathered}
\int_{0}^{t} \int_{\mathbb{R}^{n}} \tau\left(f\left(\left\|\nabla v_{m}(\tau)\right\|_{L^{2}\left(\mathbb{R}^{n}\right)}\right) \Delta v_{m}(\tau, x)-f\left(\left\|\nabla v_{l}(\tau)\right\|_{L^{2}\left(\mathbb{R}^{n}\right)}\right) \Delta v_{l}(\tau, x)\right) \times \\
\left(v_{m t}(\tau, x)-v_{l t}(\tau, x)\right) d x d \tau \\
=-\int_{0}^{t} \tau f\left(\left\|\nabla v_{m}(\tau)\right\|_{L^{2}\left(\mathbb{R}^{n}\right)}\right) \frac{d}{d \tau}\left(\left\|\nabla v_{m}(\tau)-\nabla v_{l}(\tau)\right\|_{L^{2}\left(\mathbb{R}^{n}\right)}^{2}\right) d \tau \\
+K^{m, l}(t),
\end{gathered}
$$

where

$$
\begin{gathered}
K^{m, l}(t):=\int_{0}^{t} \int_{\mathbb{R}^{n}} \tau\left(f\left(\left\|\nabla v_{m}(\tau)\right\|_{L^{2}\left(\mathbb{R}^{n}\right)}\right)-f\left(\left\|\nabla v_{l}(\tau)\right\|_{L^{2}\left(\mathbb{R}^{n}\right)}\right)\right) \times \\
\Delta v_{l}(\tau, x)\left(v_{m t}(\tau, x)-v_{l t}(\tau, x)\right) d x d \tau .
\end{gathered}
$$

By the conditions of the lemma, we obtain

$$
\limsup _{m \rightarrow \infty} \limsup _{l \rightarrow \infty}\left\|K^{m, l}\right\|_{C[0, T]}=0 .
$$

Now, let us estimate the first term on the right side of (2.1). For any $\varepsilon>0$, by integration by parts, we have

$$
\begin{aligned}
& \int_{0}^{t} \tau f\left(\left\|\nabla v_{m}(\tau)\right\|_{L^{2}\left(\mathbb{R}^{n}\right)}\right) \frac{d}{d \tau}\left(\left\|\nabla v_{m}(\tau)-\nabla v_{l}(\tau)\right\|_{L^{2}\left(\mathbb{R}^{n}\right)}^{2}\right) d \tau \\
& =\int_{0}^{t} \tau\left(f\left(\left\|\nabla v_{m}(\tau)\right\|_{L^{2}\left(\mathbb{R}^{n}\right)}\right)-f(\varepsilon)\right) \frac{d}{d \tau}\left(\left\|\nabla v_{m}(\tau)-\nabla v_{l}(\tau)\right\|_{L^{2}\left(\mathbb{R}^{n}\right)}^{2}\right) d \tau \\
& +f(\varepsilon) \int_{0}^{t} \tau \frac{d}{d \tau}\left(\left\|\nabla v_{m}(\tau)-\nabla v_{l}(\tau)\right\|_{L^{2}\left(\mathbb{R}^{n}\right)}^{2}\right) d \tau \\
& =\int_{A_{1, \varepsilon}^{m}(t)} \tau\left(f\left(\left\|\nabla v_{m}(\tau)\right\|_{L^{2}\left(\mathbb{R}^{n}\right)}\right)-f(\varepsilon)\right) \frac{d}{d \tau}\left(\left\|\nabla v_{m}(\tau)-\nabla v_{l}(\tau)\right\|_{L^{2}\left(\mathbb{R}^{n}\right)}^{2}\right) d \tau \\
& +\int_{A_{2, \varepsilon}^{m}(t)} \tau\left(f\left(\left\|\nabla v_{m}(\tau)\right\|_{L^{2}\left(\mathbb{R}^{n}\right)}\right)-f(\varepsilon)\right) \frac{d}{d \tau}\left(\left\|\nabla v_{m}(\tau)-\nabla v_{l}(\tau)\right\|_{L^{2}\left(\mathbb{R}^{n}\right)}^{2}\right) d \tau \\
& +t f(\varepsilon)\left\|\nabla v_{m}(t)-\nabla v_{l}(t)\right\|_{L^{2}\left(\mathbb{R}^{n}\right)}^{2} \\
& -f(\varepsilon) \int_{0}^{t}\left\|\nabla v_{m}(\tau)-\nabla v_{l}(\tau)\right\|_{L^{2}\left(\mathbb{R}^{n}\right)}^{2} d \tau
\end{aligned}
$$

where

$$
\begin{aligned}
& A_{1, \varepsilon}^{m}(t):=\left\{\tau \in(0, t):\left\|\nabla v_{m}(\tau)\right\|_{L^{2}\left(\mathbb{R}^{n}\right)} \leq \varepsilon\right\} \\
& A_{2, \varepsilon}^{m}(t):=\left\{\tau \in(0, t):\left\|\nabla v_{m}(\tau)\right\|_{L^{2}\left(\mathbb{R}^{n}\right)}>\varepsilon\right\} .
\end{aligned}
$$


Let us estimate the term

$$
\int_{A_{2, \varepsilon}^{m}(t)} \tau\left(f\left(\left\|\nabla v_{m}(\tau)\right\|_{L^{2}\left(\mathbb{R}^{n}\right)}\right)-f(\varepsilon)\right) \frac{d}{d \tau}\left(\left\|\nabla v_{m}(\tau)-\nabla v_{l}(\tau)\right\|_{L^{2}\left(\mathbb{R}^{n}\right)}^{2}\right) d \tau .
$$

It is enough to consider the case $A_{2, \varepsilon}^{m}(t)$ is nonempty. Since, thanks to $v_{m} \in$ $C\left([0, T] ; H^{1}\left(\mathbb{R}^{n}\right)\right)$, the set $A_{2, \varepsilon}^{m}(t)$ is open, it can be shown that it is a countable union of disjoint open intervals $\left\{\left(t_{k}, \widetilde{t}_{k}\right\}_{k=1}^{\infty}\right.$ (see, for example [22, p. 39]). Then

$$
\begin{aligned}
& \int_{A_{2, \varepsilon}^{m}(t)} \tau\left(f\left(\left\|\nabla v_{m}(\tau)\right\|_{L^{2}\left(\mathbb{R}^{n}\right)}\right)-f(\varepsilon)\right) \frac{d}{d \tau}\left(\left\|\nabla v_{m}(\tau)-\nabla v_{l}(\tau)\right\|_{L^{2}\left(\mathbb{R}^{n}\right)}^{2}\right) d \tau \\
= & \sum_{k=0}^{\infty} \int_{t_{k}}^{\tilde{t}_{k}} \tau\left(f\left(\left\|\nabla v_{m}(\tau)\right\|_{L^{2}\left(\mathbb{R}^{n}\right)}\right)-f(\varepsilon)\right) \frac{d}{d \tau}\left(\left\|\nabla v_{m}(\tau)-\nabla v_{l}(\tau)\right\|_{L^{2}\left(\mathbb{R}^{n}\right)}^{2}\right) d \tau .
\end{aligned}
$$

If $\left\|\nabla v_{m}(t)\right\|_{L^{2}\left(\mathbb{R}^{n}\right)} \leq \varepsilon$, then by continuity of $f$, we have

$$
\begin{aligned}
& f\left(\left\|\nabla v_{m}\left(t_{k}\right)\right\|_{L^{2}\left(\mathbb{R}^{n}\right)}\right)=f(\varepsilon), \quad k=1,2, \ldots, \\
& f\left(\left\|\nabla v_{m}\left(\widetilde{t}_{k}\right)\right\|_{L^{2}\left(\mathbb{R}^{n}\right)}\right)=f(\varepsilon), \quad k=1,2, \ldots .
\end{aligned}
$$

Hence, by integration by parts, we obtain

$$
\begin{aligned}
& \int_{A_{2, \varepsilon}^{m}(t)} \tau\left(f\left(\left\|\nabla v_{m}(\tau)\right\|_{L^{2}\left(\mathbb{R}^{n}\right)}\right)-f(\varepsilon)\right) \frac{d}{d \tau}\left(\left\|\nabla v_{m}(\tau)-\nabla v_{l}(\tau)\right\|_{L^{2}\left(\mathbb{R}^{n}\right)}^{2}\right) d \tau \\
& =\sum_{k=1}^{\infty} \widetilde{t}_{k}\left(f\left(\left\|\nabla v_{m}\left(\widetilde{t}_{k}\right)\right\|_{L^{2}\left(\mathbb{R}^{n}\right)}\right)-f(\varepsilon)\right)\left\|\nabla v_{m}\left(\widetilde{t}_{k}\right)-\nabla v_{l}\left(\widetilde{t}_{k}\right)\right\|_{L^{2}\left(\mathbb{R}^{n}\right)}^{2} \\
& -\sum_{k=1}^{\infty} t_{k}\left(f\left(\left\|\nabla v_{m}\left(t_{k}\right)\right\|_{L^{2}\left(\mathbb{R}^{n}\right)}\right)-f(\varepsilon)\right)\left\|\nabla v_{m}\left(t_{k}\right)-\nabla v_{l}\left(t_{k}\right)\right\|_{L^{2}\left(\mathbb{R}^{n}\right)}^{2} \\
& -\sum_{k=1}^{\infty} \int_{t_{k}}^{\tilde{t}_{k}}\left(f\left(\left\|\nabla v_{m}(\tau)\right\|_{L^{2}\left(\mathbb{R}^{n}\right)}\right)-f(\varepsilon)\right)\left\|\nabla v_{m}(\tau)-\nabla v_{l}(\tau)\right\|_{L^{2}\left(\mathbb{R}^{n}\right)}^{2} d \tau \\
& +\sum_{k=1}^{\infty} \int_{t_{k}}^{\widetilde{t}_{k}} \tau \frac{f^{\prime}\left(\left\|\nabla v_{m}(\tau)\right\|_{L^{2}\left(\mathbb{R}^{n}\right)}\right)}{\left\|\nabla v_{m}(\tau)\right\|_{L^{2}\left(\mathbb{R}^{n}\right)}}\left\langle\Delta v_{m}(\tau), v_{m t}(\tau)\right\rangle_{L^{2}\left(\mathbb{R}^{n}\right)} \times \\
& \left\|\nabla v_{m}(\tau)-\nabla v_{l}(\tau)\right\|_{L^{2}\left(\mathbb{R}^{n}\right)}^{2} d \tau \\
& =-\int_{A_{2, \varepsilon}^{m}(t)}\left(f\left(\left\|\nabla v_{m}(\tau)\right\|_{L^{2}\left(\mathbb{R}^{n}\right)}\right)-f(\varepsilon)\right)\left\|\nabla v_{m}(\tau)-\nabla v_{l}(\tau)\right\|_{L^{2}\left(\mathbb{R}^{n}\right)}^{2} d \tau \\
& +\int_{A_{2, \varepsilon}^{m}(t)} \tau \frac{f^{\prime}\left(\left\|\nabla v_{m}(\tau)\right\|_{L^{2}\left(\mathbb{R}^{n}\right)}\right)}{\left\|\nabla v_{m}(\tau)\right\|_{L^{2}\left(\mathbb{R}^{n}\right)}}\left\langle\Delta v_{m}(\tau), v_{m t}(\tau)\right\rangle_{L^{2}\left(\mathbb{R}^{n}\right)} \times \\
& \left\|\nabla v_{m}(\tau)-\nabla v_{l}(\tau)\right\|_{L^{2}\left(\mathbb{R}^{n}\right)}^{2} d \tau,
\end{aligned}
$$


where $\langle\cdot, \cdot\rangle_{L^{2}\left(\mathbb{R}^{n}\right)}$ is an inner product in $L^{2}\left(\mathbb{R}^{n}\right)$. If $\left\|\nabla v_{m}(t)\right\|_{L^{2}\left(\mathbb{R}^{n}\right)}>\varepsilon$, then the maximal element of $\left\{\widetilde{t}_{k}: k=1,2, \ldots\right\}$ is equal to $t$ and consequently, we have

$$
\begin{aligned}
& \int_{A_{2, \varepsilon}^{m}(t)} \tau\left(f\left(\left\|\nabla v_{m}(\tau)\right\|_{L^{2}\left(\mathbb{R}^{n}\right)}\right)-f(\varepsilon)\right) \frac{d}{d \tau}\left(\left\|\nabla v_{m}(\tau)-\nabla v_{l}(\tau)\right\|_{L^{2}\left(\mathbb{R}^{n}\right)}^{2}\right) d \tau \\
& =\sum_{k=1}^{\infty} \widetilde{t}_{k}\left(f\left(\left\|\nabla v_{m}\left(\widetilde{t}_{k}\right)\right\|_{L^{2}\left(\mathbb{R}^{n}\right)}\right)-f(\varepsilon)\right)\left\|\nabla v_{m}\left(\widetilde{t}_{k}\right)-\nabla v_{l}\left(\widetilde{t}_{k}\right)\right\|_{L^{2}\left(\mathbb{R}^{n}\right)}^{2} \\
& -\sum_{k=1}^{\infty} t_{k}\left(f\left(\left\|\nabla v_{m}\left(t_{k}\right)\right\|_{L^{2}\left(\mathbb{R}^{n}\right)}\right)-f(\varepsilon)\right)\left\|\nabla v_{m}\left(t_{k}\right)-\nabla v_{l}\left(t_{k}\right)\right\|_{L^{2}\left(\mathbb{R}^{n}\right)}^{2} \\
& -\sum_{k=1}^{\infty} \int_{t_{k}}^{\widetilde{t}_{k}}\left(f\left(\left\|\nabla v_{m}(\tau)\right\|_{L^{2}\left(\mathbb{R}^{n}\right)}\right)-f(\varepsilon)\right)\left\|\nabla v_{m}(\tau)-\nabla v_{l}(\tau)\right\|_{L^{2}\left(\mathbb{R}^{n}\right)}^{2} d \tau \\
& +\sum_{k=1}^{\infty} \int_{t_{k}}^{\widetilde{t}_{k}} \tau \frac{f^{\prime}\left(\left\|\nabla v_{m}(\tau)\right\|_{L^{2}\left(\mathbb{R}^{n}\right)}\right)}{\left\|\nabla v_{m}(\tau)\right\|_{L^{2}\left(\mathbb{R}^{n}\right)}}\left\langle\Delta v_{m}(\tau), v_{m t}(\tau)\right\rangle_{L^{2}\left(\mathbb{R}^{n}\right)} \times \\
& \left\|\nabla v_{m}(\tau)-\nabla v_{l}(\tau)\right\|_{L^{2}\left(\mathbb{R}^{n}\right)}^{2} d \tau \\
& =t\left(f\left(\left\|\nabla v_{m}(t)\right\|_{L^{2}\left(\mathbb{R}^{n}\right)}\right)-f(\varepsilon)\right)\left\|\nabla v_{m}(t)-\nabla v_{l}(t)\right\|_{L^{2}\left(\mathbb{R}^{n}\right)}^{2} \\
& -\int_{A_{2, \varepsilon}^{m}(t)}\left(f\left(\left\|\nabla v_{m}(\tau)\right\|_{L^{2}\left(\mathbb{R}^{n}\right)}\right)-f(\varepsilon)\right)\left\|\nabla v_{m}(\tau)-\nabla v_{l}(\tau)\right\|_{L^{2}\left(\mathbb{R}^{n}\right)}^{2} d \tau \\
& +\int_{A_{2, \varepsilon}^{m}(t)} \tau \frac{f^{\prime}\left(\left\|\nabla v_{m}(\tau)\right\|_{L^{2}\left(\mathbb{R}^{n}\right)}\right)}{\left\|\nabla v_{m}(\tau)\right\|_{L^{2}\left(\mathbb{R}^{n}\right)}}\left\langle\Delta v_{m}(\tau), v_{m t}(\tau)\right\rangle_{L^{2}\left(\mathbb{R}^{n}\right)} \times \\
& \left\|\nabla v_{m}(\tau)-\nabla v_{l}(\tau)\right\|_{L^{2}\left(\mathbb{R}^{n}\right)}^{2} d \tau .
\end{aligned}
$$

Hence, by using (1.4), (2.4) and (2.5) in (2.3), we find

$$
\begin{gathered}
-\int_{0}^{t} \tau f\left(\left\|\nabla v_{m}(\tau)\right\|_{L^{2}\left(\mathbb{R}^{n}\right)}\right) \frac{d}{d \tau}\left(\left\|\nabla v_{m}(\tau)-\nabla v_{l}(\tau)\right\|_{L^{2}\left(\mathbb{R}^{n}\right)}^{2}\right) d \tau \\
\leq f(\varepsilon) \int_{0}^{t}\left\|\nabla v_{m}(\tau)-\nabla v_{l}(\tau)\right\|_{L^{2}\left(\mathbb{R}^{n}\right)}^{2} d \tau \\
+\widehat{c}_{1} \int_{0}^{t}\left\|\nabla v_{m}(\tau)-\nabla v_{l}(\tau)\right\|_{L^{2}\left(\mathbb{R}^{n}\right)}^{2} d \tau \\
+2 \max _{s_{1}, s_{2} \epsilon[0, \varepsilon]}\left|f\left(s_{1}\right)-f\left(s_{2}\right)\right| \int_{0}^{t} \tau E\left(v_{m}(\tau)-v_{l}(\tau)\right) d \tau \\
+\frac{\widehat{c}_{1}}{\varepsilon} \int_{0}^{t} \tau E\left(v_{m}(\tau)-v_{l}(\tau)\right)\left\|v_{m t}(\tau)\right\|_{L^{2}\left(\mathbb{R}^{n}\right)} d \tau
\end{gathered}
$$




$$
\begin{gathered}
\leq\left(f(\varepsilon)+\widehat{c}_{1}\right) \int_{0}^{t}\left\|\nabla v_{m}(\tau)-\nabla v_{l}(\tau)\right\|_{L^{2}\left(\mathbb{R}^{n}\right)}^{2} d \tau \\
+\left(2 \max _{s_{1}, s_{2} \in[0, \varepsilon]}\left|f\left(s_{1}\right)-f\left(s_{2}\right)\right|+\widehat{c}_{1} \varepsilon\right) \int_{0}^{t} \tau E\left(v_{m}(\tau)-v_{l}(\tau)\right) d \tau \\
+\frac{\widehat{c}_{1}}{\varepsilon^{3}} \int_{0}^{t} \tau E\left(v_{m}(\tau)-v_{l}(\tau)\right)\left\|v_{m t}(\tau)\right\|_{L^{2}\left(\mathbb{R}^{n}\right)}^{2} d \tau, \quad \forall t \in[0, T] .
\end{gathered}
$$

Thus, setting $\gamma=2 \max _{s_{1}, s_{2} \in[0, \varepsilon]}\left|f\left(s_{1}\right)-f\left(s_{2}\right)\right|+\widehat{c}_{1} \varepsilon$ and $c_{\gamma}=\max \left\{\frac{\widehat{c}_{1}}{\varepsilon^{3}}, f(\varepsilon)+\widehat{c}_{1}\right\}$, by (2.1), (2.2) and (2.6), we get the claim of the lemma.

Now, let us prove the asymptotic compactness of $\{S(t)\}_{t \geq 0}$ in $H^{2}\left(\mathbb{R}^{n}\right) \times L^{2}\left(\mathbb{R}^{n}\right)$.

Lemma 2.2. Assume that the conditions (1.3)-(1.4) hold and $B$ is a bounded subset of $H^{2}\left(\mathbb{R}^{n}\right) \times L^{2}\left(\mathbb{R}^{n}\right)$. Then for every sequence of the form $\left\{S\left(t_{k}\right) \varphi_{k}\right\}_{k=1}^{\infty}$, where $\left\{\varphi_{k}\right\}_{k=1}^{\infty} \subset B, t_{k} \rightarrow \infty$, has a convergent subsequence in $H^{2}\left(\mathbb{R}^{n}\right) \times L^{2}\left(\mathbb{R}^{n}\right)$.

Proof. Since $\left\{\varphi_{k}\right\}_{k=1}^{\infty}$ is bounded in $H^{2}\left(\mathbb{R}^{n}\right) \times L^{2}\left(\mathbb{R}^{n}\right)$, by (1.7) it follows that the sequence $\left\{S(.) \varphi_{k}\right\}_{k=1}^{\infty}$ is bounded in $C_{b}\left(0, \infty ; H^{2}\left(\mathbb{R}^{n}\right) \times L^{2}\left(\mathbb{R}^{n}\right)\right)$, where $C_{b}\left(0, \infty ; H^{2}\left(\mathbb{R}^{n}\right) \times L^{2}\left(\mathbb{R}^{n}\right)\right)$ is the space of continuously bounded functions from $[0, \infty)$ to $H^{2}\left(\mathbb{R}^{n}\right) \times L^{2}\left(\mathbb{R}^{n}\right)$. Then for any $T \geq 0$ there exists a subsequence $\left\{k_{m}\right\}_{m=1}^{\infty}$ such that $t_{k_{m}} \geq T$, and

$$
\left\|\nabla v_{m}(t)\right\|_{L^{2}\left(\mathbb{R}^{n}\right)}^{2} \rightarrow q(t) \text { weakly star in } W^{1, \infty}(0, \infty),
$$

for some $q \in W^{1, \infty}(0, \infty)$, where $\left(v_{m}(t), v_{m t}(t)\right)=S\left(t+t_{k_{m}}-T\right) \varphi_{k_{m}}$.

Taking into account (1.3) and (1.4) in (1.6), we find

$$
\int_{0}^{T}\left\|v_{m t}(t)\right\|_{L^{2}\left(\mathbb{R}^{n}\right)}^{2} d t \leq c_{1}, \quad \forall T \geq 0 .
$$

By $(1.1)_{1}$, we have

$$
\begin{gathered}
v_{m t t}(t, x)-v_{l t t}(t, x)+\Delta^{2}\left(v_{m}(t, x)-v_{l}(t, x)\right) \\
+\alpha(x)\left(v_{m t}(t, x)-v_{l t}(t, x)\right)+\lambda\left(v_{m}(t, x)-v_{l}(t, x)\right) \\
-f\left(\left\|\nabla v_{m}(t)\right\|_{L^{2}\left(\mathbb{R}^{n}\right)}\right) \Delta v_{m}(t, x)+f\left(\left\|\nabla v_{l}(t)\right\|_{L^{2}\left(\mathbb{R}^{n}\right)}\right) \Delta v_{l}(t, x)=0 .
\end{gathered}
$$

Multiplying (2.9) by $\left(v_{m}-v_{l}\right)$ and integrating over $(0, T) \times \mathbb{R}^{n}$, we get

$$
\begin{gathered}
\int_{0}^{T}\left\|\Delta\left(v_{m}(t)-v_{l}(t)\right)\right\|_{L^{2}\left(\mathbb{R}^{n}\right)}^{2} d t+\lambda \int_{0}^{T}\left\|v_{m}(t)-v_{l}(t)\right\|_{L^{2}\left(\mathbb{R}^{n}\right)}^{2} d t \\
+\int_{0}^{T} f\left(\left\|\nabla v_{m}(t)\right\|_{L^{2}\left(\mathbb{R}^{n}\right)}\right)\left\|\nabla v_{m}(t)-\nabla v_{l}(t)\right\|_{L^{2}\left(\mathbb{R}^{n}\right)}^{2} d t \\
\leq c_{2}+c_{2} \int_{0}^{T}\left\|v_{m t}(t)-v_{l t}(t)\right\|_{L^{2}\left(\mathbb{R}^{n}\right)}^{2} d t
\end{gathered}
$$




$$
\begin{gathered}
+\int_{0}^{T}\left|f\left(\left\|\nabla v_{m}(t)\right\|_{L^{2}\left(\mathbb{R}^{n}\right)}\right)-f\left(\left\|\nabla v_{l}(t)\right\|_{L^{2}\left(\mathbb{R}^{n}\right)}\right)\right| \times \\
\left\|\nabla v_{l}(t, x)\right\|_{L^{2}\left(\mathbb{R}^{n}\right)}\left\|\nabla v_{m}(t)-\nabla v_{l}(t)\right\|_{L^{2}\left(\mathbb{R}^{n}\right)} d t .
\end{gathered}
$$

Taking into account (1.4), (2.7) and (2.8) in the last inequality and passing to the limit, we obtain

$$
\limsup _{m \rightarrow \infty} \limsup _{l \rightarrow \infty} \int_{0}^{T} E\left(v_{m}(t)-v_{l}(t)\right) d t \leq c_{3}, \quad \forall T \geq 0 .
$$

Multiplying (2.9) by $t\left(v_{m t}-v_{l t}\right)$, integrating over $(0, T) \times \mathbb{R}^{n}$ and using integration by parts, by (1.3), we find

$$
\begin{gathered}
T E\left(v_{m}(T)-v_{l}(T)\right)+\alpha_{0} \int_{0}^{T} t\left\|v_{m t}(T)-v_{l t}(T)\right\|_{L^{2}\left(\mathbb{R}^{n}\right)}^{2} d t \\
\leq \int_{0}^{T} E\left(v_{m}(t)-v_{l}(t)\right) d t \\
+\int_{0} \int_{\mathbb{R}^{n}} t\left(f\left(\left\|\nabla v_{m}(t)\right\|_{L^{2}\left(\mathbb{R}^{n}\right)}\right) \Delta v_{m}(t, x)-f\left(\left\|\nabla v_{l}(t)\right\|_{L^{2}\left(\mathbb{R}^{n}\right)}\right) \Delta v_{l}(t, x)\right) \times \\
\left(v_{m t}(t, x)-v_{l t}(t, x)\right) d x d t
\end{gathered}
$$

which, together with Lemma 2.1, gives

$$
\begin{gathered}
T E\left(v_{m}(T)-v_{l}(T)\right)+\alpha_{0} \int_{0}^{T} t\left\|v_{m t}(T)-v_{l t}(T)\right\|_{L^{2}\left(\mathbb{R}^{n}\right)}^{2} d t \\
\leq \int_{0}^{T} E\left(v_{m}(t)-v_{l}(t)\right) d t+\gamma \int_{0}^{T} t E\left(v_{m}(t)-v_{l}(t)\right) d t \\
+c_{\gamma} \int_{0}^{T} E\left(v_{m}(t)-v_{l}(t)\right) d t
\end{gathered}
$$

$$
+c_{\gamma} \int_{0}^{T} t E\left(v_{m}(t)-v_{l}(t)\right)\left\|v_{m t}(t)\right\|_{L^{2}\left(\mathbb{R}^{n}\right)}^{2} d t+K^{m, l}(T),
$$

for every $\gamma>0$. Multiplying (2.9) by $\varepsilon t\left(v_{m}-v_{l}\right)$ and integrating over $(0, T) \times \mathbb{R}^{n}$, we get

$$
\begin{aligned}
& \varepsilon \int_{0}^{T} t\left\|\Delta\left(v_{m}(t)-v_{l}(t)\right)\right\|_{L^{2}\left(\mathbb{R}^{n}\right)}^{2} d t+\varepsilon \lambda \int_{0}^{T} t\left\|v_{m}(t)-v_{l}(t)\right\|_{L^{2}\left(\mathbb{R}^{n}\right)}^{2} d t \\
& \quad \leq \varepsilon c_{3} T E\left(v_{m}(T)-v_{l}(T)\right)+\varepsilon \int_{0}^{T} t\left\|v_{m t}(t)-v_{l t}(t)\right\|_{L^{2}\left(\mathbb{R}^{n}\right)}^{2} d t
\end{aligned}
$$




$$
+\varepsilon c_{3} \int_{0}^{T}\left\|v_{m}(t)-v_{l}(t)\right\|_{L^{2}\left(\mathbb{R}^{n}\right)}^{2} d t+\varepsilon \widetilde{K}^{m, l}(T),
$$

where

$$
\begin{gathered}
\widetilde{K}^{m, l}(t):=\int_{0}^{t} \tau\left(f \left(\left\|\nabla v_{m}(\tau)\right\|_{L^{2}\left(\mathbb{R}^{n}\right)}-f\left(\left\|\nabla v_{l}(\tau)\right\|_{L^{2}\left(\mathbb{R}^{n}\right)}\right) \times\right.\right. \\
\left\|\nabla v_{l}(\tau)\right\|_{L^{2}\left(\mathbb{R}^{n}\right)}\left\|v_{m}(\tau)-v_{l}(\tau)\right\|_{L^{2}\left(\mathbb{R}^{n}\right)} d \tau,
\end{gathered}
$$

and by (1.4) and (2.7), it is easy to see that

$$
\limsup _{m \rightarrow \infty} \limsup _{l \rightarrow \infty}\left\|\widetilde{K}^{m, l}\right\|_{C[0, T]}=0 .
$$

Adding (2.11) to (2.12) and choosing $\gamma$ and $\varepsilon$ small enough, we obtain

$$
\begin{gathered}
T E\left(v_{m}(T)-v_{l}(T)\right) \leq c_{4} \int_{0}^{T} t E\left(v_{m}(t)-v_{l}(t)\right)\left\|v_{m t}(t)\right\|_{L^{2}\left(\mathbb{R}^{n}\right)}^{2} d t \\
+c_{4} \int_{0}^{T} E\left(v_{m}(t)-v_{l}(t)\right) d t+c_{4}\left|K^{m, l}(T)\right|+c_{4}\left|\widetilde{K}^{m, l}(T)\right|, \quad \forall T \geq 0 .
\end{gathered}
$$

Now, denoting $y_{m, l}(t):=t E\left(v_{m}(t)-v_{l}(t)\right)$ and applying Gronwall inequality, we get

$$
\begin{gathered}
y_{m, l}(T) \\
\leq c_{4}\left(\int_{0}^{T} E\left(v_{m}(t)-v_{l}(t)\right) d t+\left\|K^{m, l}\right\|_{C[0, T]}+\left\|\widetilde{K}^{m, l}\right\|_{C[0, T]}\right) \times \\
e^{\int^{T}\left\|v_{m t}(t)\right\|_{L^{2}\left(\mathbb{R}^{n}\right)}^{2} d t},
\end{gathered}
$$

which, together with (2.8), yields

$$
\begin{gathered}
T E\left(v_{m}(T)-v_{l}(T)\right) \\
\leq c_{5}\left(\int_{0}^{T} E\left(v_{m}(t)-v_{l}(t)\right) d t+\left\|K^{m, l}\right\|_{C[0, T]}+\left\|\widetilde{K}^{m, l}\right\|_{C[0, T]}\right),
\end{gathered}
$$

for every $T \geq 0$. Passing to the limit in the above inequality and taking into account (2.10), we find

$$
\limsup _{m \rightarrow \infty} \limsup _{l \rightarrow \infty} T E\left(v_{m}(T)-v_{l}(T)\right) \leq c_{6}, \quad \forall T \geq 0,
$$

which gives

$$
\limsup _{m \rightarrow \infty} \limsup _{l \rightarrow \infty}\left\|S\left(t_{k_{m}}\right) \varphi_{k_{m}}-S\left(t_{k_{l}}\right) \varphi_{k_{l}}\right\|_{H^{2}\left(\mathbb{R}^{n}\right) \times L^{2}\left(\mathbb{R}^{n}\right)} \leq \frac{c_{7}}{\sqrt{T}}, \quad \forall T>0 .
$$

Consequently, we have

$$
\liminf _{l \rightarrow \infty} \liminf _{m \rightarrow \infty}\left\|S\left(t_{k}\right) \varphi_{k}-S\left(t_{m}\right) \varphi_{m}\right\|_{H^{2}\left(\mathbb{R}^{n}\right) \times L^{2}\left(\mathbb{R}^{n}\right)}=0 .
$$

Thus, by using the argument at the end of the proof of [23, Lemma 3.4], we complete the proof of the lemma. 
Since, by (1.3) and (1.6), problem (1.1)-(1.2) admidts a strict Lyapunov function

$$
\Phi(u(t))=E(u(t))+\frac{1}{2} F\left(\|\nabla u(t)\|_{L^{2}\left(\mathbb{R}^{n}\right)}^{2}\right)-\int_{\mathbb{R}^{n}} h(x) u(t, x) d x,
$$

applying [20, Corollary 7.5.7], we have the following theorem.

THEOREM 2.1. Under conditions (1.3)-(1.6) the semigroup $\{S(t)\}_{t>0}$ generated by the problem (1.1)-(1.2) possesses a global attractor $\mathcal{A}$ in $H^{2}\left(\mathbb{R}^{n}\right) \times L^{2}\left(\mathbb{R}^{n}\right)$ and $\mathcal{A}=\mathcal{M}^{u}(\mathcal{N})$.

\section{Regularity of the global attractor} lemma.

To prove the regularity of the global attractor, we start with the following

LEMMA 3.1. Assume that the conditions (1.3) and (1.4) hold and $B$ is a bounded subset in $H^{4}\left(\mathbb{R}^{n}\right) \times H^{2}\left(\mathbb{R}^{n}\right)$. Then there exists a constant $C>0$ such that

$$
\sup _{\varphi \in B}\|S(t) \varphi\|_{H^{4}\left(\mathbb{R}^{n}\right) \times H^{2}\left(\mathbb{R}^{n}\right)} \leq C,
$$

for all $t \geq 0$.

Proof. Let $\left(u_{0}, u_{1}\right) \in B$ and $\left(u(t), u_{t}(t)\right):=S(t)\left(u_{0}, u_{1}\right)$. Then, as mentioned in Introduction,

$$
u \in C\left([0, \infty) ; H^{4}\left(\mathbb{R}^{n}\right)\right) \cap C^{1}\left([0, \infty) ; H^{2}\left(\mathbb{R}^{n}\right)\right) \cap C^{2}\left([0, \infty) ; L^{2}\left(\mathbb{R}^{n}\right)\right)
$$

is the strong solution of (1.1). Defining

$$
v(t, x):=\frac{u(t+\tau, x)-u(t, x)}{\tau}, \tau>0
$$

by (1.1), we get

$$
\begin{gathered}
v_{t t}(t, x)+\Delta^{2} v(t, x)+\alpha(x) v_{t}(t, x) \\
+\lambda v(t, x)-f\left(\|\nabla u(t)\|_{L^{2}\left(\mathbb{R}^{n}\right)}\right) \Delta v(t, x) \\
-\frac{f\left(\|\nabla u(t+\tau)\|_{L^{2}\left(\mathbb{R}^{n}\right)}\right)-f\left(\|\nabla u(t)\|_{L^{2}\left(\mathbb{R}^{n}\right)}\right)}{\tau} \times \\
\Delta u(t+\tau, x)=0, \quad(t, x) \in(0, \infty) \times \mathbb{R}^{n} .
\end{gathered}
$$

Multiplying (3.1) by $v_{t}$ and integrating over $\mathbb{R}^{n}$, we find

$$
\begin{gathered}
\frac{d}{d t} E(v(t))+\int_{\mathbb{R}^{n}} \alpha(x)\left|v_{t}(t, x)\right|^{2} d x \\
+\frac{1}{2} f\left(\|\nabla u(t)\|_{L^{2}\left(\mathbb{R}^{n}\right)}\right) \frac{d}{d t}\left(\|\nabla v(t)\|_{L^{2}\left(\mathbb{R}^{n}\right)}^{2}\right) \\
-\frac{f\left(\|\nabla u(t+\tau)\|_{L^{2}\left(\mathbb{R}^{n}\right)}\right)-f\left(\|\nabla u(t)\|_{L^{2}\left(\mathbb{R}^{n}\right)}\right)}{\tau} \times \\
\int_{\mathbb{R}^{n}} \Delta u(t+\tau, x) v_{t}(t, x) d x=0 .
\end{gathered}
$$


Since

$$
\begin{gathered}
\left|\frac{d}{d t} f\left(\|\nabla u(t)\|_{L^{2}\left(\mathbb{R}^{n}\right)}\right)\right|=\left|\frac{f^{\prime}\left(\|\nabla u(t)\|_{L^{2}\left(\mathbb{R}^{n}\right)}\right)}{\|\nabla u(t)\|_{L^{2}\left(\mathbb{R}^{n}\right)}}\left\langle\nabla u(t), \nabla u_{t}(t)\right\rangle_{L^{2}\left(\mathbb{R}^{n}\right)}\right| \\
\leq\left|f^{\prime}\left(\|\nabla u(t)\|_{L^{2}\left(\mathbb{R}^{n}\right)}\right)\right|\left\|\nabla u_{t}(t)\right\|_{L^{2}\left(\mathbb{R}^{n}\right)}, \text { a.e. in }(0, \infty),
\end{gathered}
$$

considering (1.3) and (1.7) in (3.2), we obtain

$$
\begin{aligned}
\frac{d}{d t}(E(v(t))+ & \left.\frac{1}{2} f\left(\|\nabla u(t)\|_{L^{2}\left(\mathbb{R}^{n}\right)}\right)\|\nabla v(t)\|_{L^{2}\left(\mathbb{R}^{n}\right)}^{2}\right)+\alpha_{0}\left\|v_{t}(t)\right\|_{L^{2}\left(\mathbb{R}^{n}\right)}^{2} \\
\leq & c_{1}\left(\left\|\nabla u_{t}(t)\right\|_{L^{2}\left(\mathbb{R}^{n}\right)}\|\nabla v(t)\|_{L^{2}\left(\mathbb{R}^{n}\right)}^{2}\right. \\
+ & \left.\|\nabla v(t)\|_{L^{2}\left(\mathbb{R}^{n}\right)} \int_{\mathbb{R}^{n}} \Delta u(t+\tau, x) v_{t}(t, x) d x\right) \\
\leq & c_{2}\left(\left\|\nabla u_{t}(t)\right\|_{L^{2}\left(\mathbb{R}^{n}\right)}\|v(t)\|_{H^{2}\left(\mathbb{R}^{n}\right)}\|v(t)\|_{L^{2}\left(\mathbb{R}^{n}\right)}\right. \\
+ & \left.\|v(t)\|_{H^{2}\left(\mathbb{R}^{n}\right)}^{\frac{1}{2}}\|v(t)\|_{L^{2}\left(\mathbb{R}^{n}\right)}^{\frac{1}{2}}\left\|v_{t}(t)\right\|_{L^{2}\left(\mathbb{R}^{n}\right)}\right) .
\end{aligned}
$$

Since, by (1.7),

$$
\|v(t)\|_{L^{2}\left(\mathbb{R}^{n}\right)}=\left\|\frac{u(t+\tau, x)-u(t, x)}{\tau}\right\|_{L^{2}\left(\mathbb{R}^{n}\right)} \leq \sup _{0 \leq t<\infty}\left\|u_{t}(t)\right\|_{L^{2}\left(\mathbb{R}^{n}\right)}<\widehat{C},
$$

by the previous inequality, we get

$$
\begin{aligned}
\frac{d}{d t}\left(E(v(t))+\frac{1}{2} f\right. & \left.\left(\|\nabla u(t)\|_{L^{2}\left(\mathbb{R}^{n}\right)}\right)\|\nabla v(t)\|_{L^{2}\left(\mathbb{R}^{n}\right)}^{2}\right)+\alpha_{0}\left\|v_{t}(t)\right\|_{L^{2}\left(\mathbb{R}^{n}\right)}^{2} \\
\leq & c_{3}\left(\left\|\nabla u_{t}(t)\right\|_{L^{2}\left(\mathbb{R}^{n}\right)}\|v(t)\|_{H^{2}\left(\mathbb{R}^{n}\right)}\right. \\
& \left.+\|v(t)\|_{H^{2}\left(\mathbb{R}^{n}\right)}^{\frac{1}{2}}\left\|v_{t}(t)\right\|_{L^{2}\left(\mathbb{R}^{n}\right)}\right) .
\end{aligned}
$$

Multiplying (3.1) by $\varepsilon v$ and integrating over $\mathbb{R}^{n}$, we find

$$
\begin{gathered}
\varepsilon \frac{d}{d t}\left(\left\langle\nabla v(t), \nabla v_{t}(t)\right\rangle_{L^{2}\left(\mathbb{R}^{n}\right)}+\frac{1}{2} \int_{\mathbb{R}^{n}} \alpha(x) v(t, x)^{2} d x\right) \\
+\varepsilon\|\Delta v(t)\|_{L^{2}\left(\mathbb{R}^{n}\right)}^{2}+\varepsilon \lambda\|v(t)\|_{L^{2}\left(\mathbb{R}^{n}\right)}^{2} \\
\leq \varepsilon\left\|v_{t}(t)\right\|_{L^{2}\left(\mathbb{R}^{n}\right)}^{2}+\varepsilon c_{4}\|v(t)\|_{H^{1}\left(\mathbb{R}^{n}\right)} .
\end{gathered}
$$

Considering (3.3) and (3.4), for sufficiently small $\varepsilon>0$ and applying Young inequality, we obtain

$$
\frac{d}{d t} \Psi(t)+c_{5} E(v(t)) \leq c_{6}+c_{6}\left\|\nabla u_{t}(t)\right\|_{L^{2}\left(\mathbb{R}^{n}\right)}^{2},
$$

where $c_{5}>0$ and

$$
\begin{gathered}
\Psi(t):=E(v(t))+\frac{1}{2} f(\|\nabla u(t)\|)\|\nabla v(t)\|_{L^{2}\left(\mathbb{R}^{n}\right)}^{2} \\
+\varepsilon\left\langle\nabla v(t), \nabla v_{t}(t)\right\rangle_{L^{2}\left(\mathbb{R}^{n}\right)}+\frac{\varepsilon}{2} \int_{\mathbb{R}^{n}} \alpha(x)|v(t, x)|^{2} d x .
\end{gathered}
$$


Since $\varepsilon>0$ is sufficiently small, there exist constants $c>0, \widetilde{c}>0$ such that

$$
c E(v(t)) \leq \Psi(t) \leq \widetilde{c} E(v(t)) .
$$

Then, by (3.5) and (3.6), we have

which yields

$$
\frac{d}{d t} \Psi(t)+c_{7} \Psi(t) \leq c_{6}+c_{6}\left\|\nabla u_{t}(t)\right\|_{L^{2}\left(\mathbb{R}^{n}\right)}^{2},
$$

$$
\begin{aligned}
& \Psi(t) \leq c_{8}+e^{-c_{7} t} c_{8} \int_{0}^{t}\left\|\nabla u_{t}(s)\right\|_{L^{2}\left(\mathbb{R}^{n}\right)}^{2} e^{c_{7} s} d s \\
& \leq c_{8}+c_{8} \sup _{t \in[0, T]}\left(\left\|u_{t}(t)\right\|_{L^{2}\left(\mathbb{R}^{n}\right)}\left\|u_{t}(t)\right\|_{H^{2}\left(\mathbb{R}^{n}\right)}\right),
\end{aligned}
$$

for every $T \geq 0$. Taking into account (1.7) and (3.6) in the last inequality, we find

$$
E(v(t)) \leq c_{9}+c_{9} \sup _{t \in[0, T]}\left\|u_{t}(t)\right\|_{H^{2}\left(\mathbb{R}^{n}\right)}, \quad \forall t \in[0, T],
$$

for every $T \geq 0$. Passing to limit as $\tau \rightarrow 0$ in the above inequality, from the definition of $v$, we obtain

$$
E\left(u_{t}(t)\right) \leq c_{9}+c_{9} \sup _{t \in[0, T]}\left\|u_{t}(t)\right\|_{H^{2}\left(\mathbb{R}^{n}\right)}, \quad \forall t \in[0, T]
$$

for every $T \geq 0$. Thus, after taking supremum on $[0, T]$ and applying Young inequality, we have

$$
E\left(u_{t}(t)\right) \leq c_{10}, \quad \forall t \geq 0
$$

Taking into account this estimate in (1.1), we find that

$$
\|u(t)\|_{H^{4}\left(\mathbb{R}^{n}\right)} \leq c_{11},
$$

which, together with previous inequality, yields

$$
\left\|\left(u(t), u_{t}(t)\right)\right\|_{H^{4}\left(\mathbb{R}^{n}\right) \times H^{2}\left(\mathbb{R}^{n}\right)} \leq C,
$$

for some constant $C>0$.

Now we can show the regularity of the attractor.

THEOREM 3.1. Under the assumptions of Theorem 1.1, the global attractor $\mathcal{A}$ for the problem (1.1)-(1.2) is bounded in $H^{4}\left(\mathbb{R}^{n}\right) \times H^{2}\left(\mathbb{R}^{n}\right)$.

Proof. Let $\theta \in \mathcal{A}$. By the invariance of $\mathcal{A}$, it follows that (see [24, p. 159]) there exists an invariant trajectory $\gamma=\left\{U(t)=\left(u(t), u_{t}(t)\right): t \in \mathbb{R}\right\} \subset \mathcal{A}$ such that $U(0)=\theta$. By an invariant trajectory we mean a curve $\gamma=\{U(t): t \in \mathbb{R}\}$ such that $S(t) U(\tau)=U(t+\tau)$ for all $t \geq 0$ and $\tau \in \mathbb{R}$ (see [24, p. 157])

In the case when $h \equiv 0$ in equation (1.1), by (1.4), it follows that the stationary point set $\mathcal{N}=\{(0,0)\}$. By Theorem 1.1 and the definition of unstable manifold, we have

$$
\lim _{t \rightarrow-\infty} \inf _{w \in \mathcal{N}}\|U(t)-w\|_{H^{2}\left(\mathbb{R}^{n}\right) \times L^{2}\left(\mathbb{R}^{n}\right)}=0 .
$$

Then, from the monotonicity of the Lyapunov function $\Phi(\cdot)$, we have $\mathcal{A}=\{(0,0)\}$.

So, we will consider the case $h \neq 0$. In this case, it is clear that $\mathcal{N}$ does not contain $(0,0)$. Since $\mathcal{N}$ is compact (because it is a closed subset of $\mathcal{A}$ ), we obtain

$$
\min _{(\varphi, 0) \in \mathcal{N}}\|\nabla \varphi\|_{L^{2}\left(\mathbb{R}^{n}\right)} \geq c_{0}
$$


for some $c_{0}>0$. This inequality, together with (3.7), yields that there exists $t_{0} \in(-\infty, 0)$ such that

$$
\|\nabla u(t)\|_{L^{2}\left(\mathbb{R}^{n}\right)} \geq \frac{c_{0}}{2}, \forall t \leq t_{0} .
$$

Now, again defining

$$
v(t, x):=\frac{u(t+\tau, x)-u(t, x)}{\tau},
$$

we have equation (3.1). Multiplying (3.1) by $v_{t}$ and integrating over $\mathbb{R}^{n}$, by using (3.8), we get

$$
\begin{gathered}
\frac{d}{d t}\left(E(v(t))+\frac{1}{2} f(\|\nabla u(t)\|)\|\nabla v(t)\|_{L^{2}\left(\mathbb{R}^{n}\right)}^{2}\right)+\alpha_{0}\left\|v_{t}(t)\right\|_{L^{2}\left(\mathbb{R}^{n}\right)}^{2} \\
\leq \frac{f^{\prime}(\|\nabla u(t)\|)}{\|\nabla u(t)\|}\|\Delta u(t)\|\left\|u_{t}(t)\right\|\|\nabla v(t)\|_{L^{2}\left(\mathbb{R}^{n}\right)}^{2} \\
+\widehat{c}_{1}\|\nabla v(t)\|_{L^{2}\left(\mathbb{R}^{n}\right)} \int_{\mathbb{R}^{n}} \Delta u(t+\tau, x) v_{t}(t, x) d x \\
\leq \widehat{c}_{2}\|\nabla v(t)\|_{L^{2}\left(\mathbb{R}^{n}\right)}^{2}+\widehat{c}_{2}\|\nabla v(t)\|_{L^{2}\left(\mathbb{R}^{n}\right)}\left\|v_{t}(t)\right\|_{L^{2}\left(\mathbb{R}^{n}\right)} \\
\leq \widehat{c}_{3}\|v(t)\|_{H^{2}\left(\mathbb{R}^{n}\right)}+\widehat{c}_{3}\|v(t)\|_{H^{2}\left(\mathbb{R}^{n}\right)}^{\frac{1}{2}}\left\|v_{t}(t)\right\|_{L^{2}\left(\mathbb{R}^{n}\right)} .
\end{gathered}
$$

By using (3.4), (3.6) and (3.9), we find

$$
\frac{d}{d t} \Psi(t)+\widehat{c}_{4} \Psi(t) \leq \widehat{c}_{5}, \quad \forall t \leq t_{0},
$$

which yields

$$
\Psi(t) \leq \widehat{c}_{6}+e^{\widehat{c}_{4}(s-t)} \Psi(s), \quad s \leq t \leq t_{0},
$$

where $\widehat{c}_{4}>0$. Then, passing to limit as $s \rightarrow-\infty$ and taking into account that $\cup_{t \in \mathbb{R}} U(t) \subset \mathcal{A}$, we have

$$
\Psi(t) \leq \widehat{c}_{6}
$$

which, by (3.6), gives

$$
E(v(t)) \leq \widehat{c}_{7} .
$$

Now, passing to limit as $\tau \rightarrow 0$ in the last inequality, we obtain

$$
E\left(u_{t}(t)\right) \leq \widehat{c}_{7}, \quad \forall t \leq t_{0} .
$$

Considering (3.10) in (1.1), we find

$$
\|u(t)\|_{H^{4}\left(\mathbb{R}^{n}\right)} \leq \widehat{c}_{8}, \quad \forall t \leq t_{0},
$$

which, together with (3.10), yields

$$
\left\|\left(u(t), u_{t}(t)\right)\right\|_{H^{4}\left(\mathbb{R}^{n}\right) \times H^{2}\left(\mathbb{R}^{n}\right)} \leq \widehat{c}_{9}, \quad \forall t \leq t_{0} .
$$

Thus, applying Lemma 3.1 to the set $B=\left\{\left(u(t), u_{t}(t)\right): t \in\left(-\infty, t_{0}\right]\right\}$, we obtain

$$
\|\theta\|_{H^{4}\left(\mathbb{R}^{n}\right) \times H^{2}\left(\mathbb{R}^{n}\right)} \leq C,
$$

where $C>0$ is a constant independent of $\theta$. 


\section{Finite dimensionality of the global attractor}

In this section, we will use the idea of the [14] to obtain the finite dimensionality. Let us start with the following lemma.

Lemma 4.1. Assume that the conditions (1.3) and (1.4) hold and

$$
u \in W^{1, \infty}\left(0, \infty ; H^{2}\left(\mathbb{R}^{n}\right)\right)
$$

such that

$$
\|u\|_{W^{1, \infty}\left(0, \infty ; H^{2}\left(\mathbb{R}^{n}\right)\right)}+\int_{0}^{\infty}\left\|u_{t}(t)\right\|_{L^{2}\left(\mathbb{R}^{n}\right)}^{2} d t<c,
$$

for some constant $c>0$. Also, let $\{T(t, \tau)\}_{t \geq \tau}$ be the process generated by the problem

$$
\left\{\begin{array}{l}
v_{t t}+\Delta^{2} v+\alpha(x) v_{t}+\lambda v \\
\quad-f\left(\|\nabla u(t)\|_{L^{2}\left(\mathbb{R}^{n}\right)}\right) \Delta v=0, \quad t \geq \tau \\
v(\tau)=v_{0}, \quad v_{t}(\tau)=v_{1}, \quad \tau \geq 0
\end{array}\right.
$$

in $H^{2}\left(\mathbb{R}^{n}\right) \times L^{2}\left(\mathbb{R}^{n}\right)$. Then there exist $M=M(c)>1$ and $\omega=\omega(c)>0$ such that

$$
\|T(t, \tau)\|_{L\left(H^{2(1+i)}\left(\mathbb{R}^{n}\right) \times H^{2 i}\left(\mathbb{R}^{n}\right)\right)} \leq M e^{-\omega(t-\tau)}, \quad \forall t \geq \tau,
$$

where $i=0,1$ and $L(X)$ is the space of linear bounded operators in $X$.

Proof. By using the multiplier $\left(v_{t}+\varepsilon v\right)$ as in Lemma 3.1 , for sufficiently small $\varepsilon>0$ and applying Young inequality, we get

$$
\frac{d}{d t} \Psi(t)+\gamma E(v(t)) \leq c_{1}\left\|\nabla u_{t}(t)\right\|_{L^{2}\left(\mathbb{R}^{n}\right)}\|\nabla v(t)\|_{L^{2}\left(\mathbb{R}^{n}\right)}^{2},
$$

for some $\gamma>0$. Then, by using interpolation, (3.6) and (4.1), we find

$$
\frac{d}{d t} \Psi(t)+\gamma \Psi(v(t)) \leq c_{2}\left\|u_{t}(t)\right\|_{L^{2}\left(\mathbb{R}^{n}\right)}^{\frac{1}{2}} \Psi(t) .
$$

Hence, by Gronwall inequality and (3.6), we have,

$$
E(t) \leq c_{3} E(\tau) e^{c_{2} \int_{\tau}^{t}\left\|u_{t}(\sigma)\right\|_{L^{2}\left(\mathbb{R}^{n}\right)}^{\frac{1}{2}} d \sigma-\gamma(t-\tau)} .
$$

Since, by Holder inequality and (4.1),

$$
\int_{\tau}^{t}\left\|u_{t}(\sigma)\right\|_{L^{2}\left(\mathbb{R}^{n}\right)}^{\frac{1}{2}} d \sigma \leq c_{4}(t-\tau)^{\frac{3}{4}},
$$

from (4.3) it follows that

$$
\|T(t, \tau)\|_{L\left(H^{2}\left(\mathbb{R}^{n}\right) \times L^{2}\left(\mathbb{R}^{n}\right)\right)} \leq M_{1} e^{-\omega(t-\tau)}, \quad \forall t \geq \tau,
$$

for some $M_{1}>1$ and $\omega>0$.

Now, let us define $w:=v_{t}$. Then $w$ is the solution of the following equation

$$
\begin{gathered}
w_{t t}(t, x)+\Delta^{2} w(t, x)+\alpha(x) w_{t}(t, x) \\
+\lambda w(t, x)-f\left(\|\nabla u(t)\|_{L^{2}\left(\mathbb{R}^{n}\right)}\right) \Delta w(t, x) \\
-\frac{f^{\prime}\left(\|\nabla u(t)\|_{L^{2}\left(\mathbb{R}^{n}\right)}\right)}{\|\nabla u(t)\|_{L^{2}\left(\mathbb{R}^{n}\right)}}\left\langle\nabla u_{t}(t), \nabla u(t)\right\rangle_{L^{2}\left(\mathbb{R}^{n}\right)} \Delta v(t, x)=0, \quad t \geq \tau, x \in \mathbb{R}^{n},
\end{gathered}
$$


and by the variation of parameters formula, we have

$$
W(t)=T(t, \tau) W(\tau)+\int_{\tau}^{t} T(t, s) G(s) d s,
$$

where $W(t):=\left(w(t), w_{t}(t)\right)$ and

$G(t):=\left(0, \frac{f^{\prime}\left(\|\nabla u(t)\|_{L^{2}\left(\mathbb{R}^{n}\right)}\right)}{\|\nabla u(t)\|_{L^{2}\left(\mathbb{R}^{n}\right)}}\left\langle\nabla u_{t}(t), \nabla u(t)\right\rangle_{L^{2}\left(\mathbb{R}^{n}\right)} \Delta v(t)\right)$. Therefore, by using (4.4), we find

$$
\begin{gathered}
\|W(t)\|_{H^{2}\left(\mathbb{R}^{n}\right) \times L^{2}\left(\mathbb{R}^{n}\right)} \leq\|T(t, \tau) W(\tau)\|_{H^{2}\left(\mathbb{R}^{n}\right) \times L^{2}\left(\mathbb{R}^{n}\right)} \\
+\int_{\tau}^{t}\|T(t, s) G(s)\|_{H^{2}\left(\mathbb{R}^{n}\right) \times L^{2}\left(\mathbb{R}^{n}\right)} d s \\
\leq M_{1} e^{-\omega(t-\tau)}\|W(\tau)\|_{H^{2}\left(\mathbb{R}^{n}\right) \times L^{2}\left(\mathbb{R}^{n}\right)}+c_{5} \int_{\tau}^{t} e^{-\omega(t-s)}\|G(s)\|_{H^{2}\left(\mathbb{R}^{n}\right) \times L^{2}\left(\mathbb{R}^{n}\right)} d s \\
\leq M_{1} e^{-\omega(t-\tau)}\|W(\tau)\|_{H^{2}\left(\mathbb{R}^{n}\right) \times L^{2}\left(\mathbb{R}^{n}\right)}+c_{6} \int_{\tau}^{t} e^{-\omega(t-s)}\|v(s)\|_{H^{2}\left(\mathbb{R}^{n}\right)} d s \\
\leq M_{1} e^{-\omega(t-\tau)}\|W(\tau)\|_{H^{2}\left(\mathbb{R}^{n}\right) \times L^{2}\left(\mathbb{R}^{n}\right)} \\
+c_{7} \int_{\tau}^{t} e^{-\omega(t-s)} e^{-\omega(s-\tau)}\left\|\left(v(\tau), v_{t}(\tau)\right)\right\|_{H^{2}\left(\mathbb{R}^{n}\right) \times L^{2}\left(\mathbb{R}^{n}\right)} d s \\
\leq c_{8} e^{-\omega(t-\tau)}\left(\|W(\tau)\|_{H^{2}\left(\mathbb{R}^{n}\right) \times L^{2}\left(\mathbb{R}^{n}\right)}+\left\|\left(v(\tau), v_{t}(\tau)\right)\right\|_{H^{2}\left(\mathbb{R}^{n}\right) \times L^{2}\left(\mathbb{R}^{n}\right)}\right), \forall t \geq \tau .
\end{gathered}
$$

Thus, the last inequality, together with $(4.2)_{1}$, gives

$$
\|T(t, \tau)\|_{L\left(H^{4}\left(\mathbb{R}^{n}\right) \times H^{2}\left(\mathbb{R}^{n}\right)\right)} \leq M_{2} e^{-\omega(t-\tau)}, \quad \forall t \geq \tau,
$$

for some $M_{2}>1$.

Now, we can give the theorem about the finite dimensionality of the global attractor.

TheOREm 4.1. The fractal dimension of the global attractor $\mathcal{A}$ is finite.

Proof. Let $\theta_{1}, \theta_{2} \in \mathcal{A}$ and $\left(u(t), u_{t}(t)\right)=S(t) \theta_{1},\left(v(t), v_{t}(t)\right)=S(t) \theta_{2}$. Define $w(t):=v(t)-u(t)$. Then, we find

$$
\begin{gathered}
w_{t t}(t, x)+\Delta^{2} w(t, x)+\alpha(x) w_{t}(t, x) \\
+\lambda w(t, x)-f\left(\|\nabla u(t)\|_{L^{2}\left(\mathbb{R}^{n}\right)}\right) \Delta w(t, x) \\
-\left(f\left(\|\nabla v(t)\|_{L^{2}\left(\mathbb{R}^{n}\right)}\right)-f\left(\|\nabla u(t)\|_{L^{2}\left(\mathbb{R}^{n}\right)}\right)\right) \Delta v(t, x)=0 .
\end{gathered}
$$

Hence, by the variation of parameters formula, we have

$$
\left(w(t), w_{t}(t)\right)=T(t, 0)\left(w(0), w_{t}(0)\right)+\int_{0}^{t} T(t, \tau) \widehat{G}(\tau) d \tau
$$


where $\widehat{G}(t)=\left(0,\left(f\left(\|\nabla v(t)\|_{L^{2}\left(\mathbb{R}^{n}\right)}\right)-f\left(\|\nabla u(t)\|_{L^{2}\left(\mathbb{R}^{n}\right)}\right)\right) \Delta v(t)\right)$. By Lemma 4.1, we get

$$
\begin{gathered}
\left\|S(t) \theta_{2}-S(t) \theta_{1}\right\|_{H^{4}\left(\mathbb{R}^{n}\right) \times H^{2}\left(\mathbb{R}^{n}\right)} \\
\leq M e^{-\omega t}\left\|\theta_{2}-\theta_{1}\right\|_{H^{4}\left(\mathbb{R}^{n}\right) \times H^{2}\left(\mathbb{R}^{n}\right)} \\
+\widetilde{c}_{1} \int_{0}^{t} e^{-\omega(t-\tau)}\left\|S(\tau) \theta_{2}-S(\tau) \theta_{1}\right\|_{H^{2}\left(\mathbb{R}^{n}\right) \times L^{2}\left(\mathbb{R}^{n}\right)} d \tau .
\end{gathered}
$$

Applying Gronwall lemma to (4.6), we obtain

$$
\begin{gathered}
\left\|S(t) \theta_{2}-S(t) \theta_{1}\right\|_{H^{4}\left(\mathbb{R}^{n}\right) \times H^{2}\left(\mathbb{R}^{n}\right)} \\
\leq M e^{\left(\widetilde{c}_{2}-\omega\right) t}\left\|\theta_{2}-\theta_{1}\right\|_{H^{4}\left(\mathbb{R}^{n}\right) \times H^{2}\left(\mathbb{R}^{n}\right)}, \quad \forall t \geq 0 .
\end{gathered}
$$

Also, by (4.6), we have

$$
\begin{gathered}
\left\|S(t) \theta_{2}-S(t) \theta_{1}\right\|_{H^{4}\left(\mathbb{R}^{n}\right) \times H^{2}\left(\mathbb{R}^{n}\right)} \\
\leq M e^{-\omega t}\left\|\theta_{2}-\theta_{1}\right\|_{H^{4}\left(\mathbb{R}^{n}\right) \times H^{2}\left(\mathbb{R}^{n}\right)} \\
+\frac{\widetilde{c}_{1}}{\omega} \sup _{0 \leq \tau \leq t}\left\|S(\tau) \theta_{2}-S(\tau) \theta_{1}\right\|_{H^{2}(B(0, r)) \times L^{2}(B(0, r))}+\widetilde{c}_{1} \int_{0}^{t} e^{-\omega(t-\tau)} \times \\
\left\|S(\tau) \theta_{2}-S(\tau) \theta_{1}\right\|_{H^{2}\left(\mathbb{R}^{n} \backslash B(0, r)\right) \times L^{2}\left(\mathbb{R}^{n} \backslash B(0, r)\right)} d \tau, \quad \forall t \geq 0, \forall r>0,
\end{gathered}
$$

where $B(0, r)=\left\{x: x \in \mathbb{R}^{n},|x|<r\right\}$.

Now, we will estimate the integral term on the right hand side of (4.8). Let

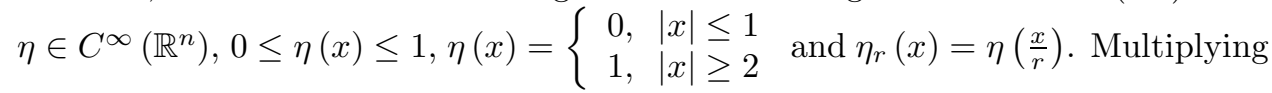
(4.5) by $\eta_{r}$ and denoting $w_{r}(t)=\eta_{r} w(t)$, we get

$$
\begin{gathered}
w_{r t t}(t, x)+\Delta^{2} w_{r}(t, x)+\alpha(x) w_{r t}(t, x) \\
+\lambda w_{r}(t, x)-f\left(\|\nabla u(t)\|_{L^{2}\left(\mathbb{R}^{n}\right)}\right) \Delta w_{r}(t, x) \\
-\eta_{r}\left(f\left(\|\nabla v(t)\|_{L^{2}\left(\mathbb{R}^{n}\right)}\right)-f\left(\|\nabla u(t)\|_{L^{2}\left(\mathbb{R}^{n}\right)}\right)\right) \Delta v(t, x)=f_{1}(t),
\end{gathered}
$$

where

$$
\begin{gathered}
f_{1}(t)=\Delta^{2} \eta_{r} w+2 \Delta \eta_{r} \Delta w+2 \sum_{i=1}^{n}\left(\Delta \eta_{r}\right)_{x_{i}} w_{x_{i}} \\
+2 \sum_{i=1}^{n}\left(\eta_{r}\right)_{x_{i}} \Delta w_{x_{i}}+4 \sum_{i, j=1}^{n}\left(\eta_{r}\right)_{x_{i} x_{j}} w_{x_{i} x_{j}} \\
-\Delta \eta_{r} f\left(\|\nabla u(t)\|_{L^{2}\left(\mathbb{R}^{n}\right)}\right) w-2 f\left(\|\nabla u(t)\|_{L^{2}\left(\mathbb{R}^{n}\right)}\right) \sum_{i=1}^{n}\left(\eta_{r}\right)_{x_{i}} w_{x_{i}} .
\end{gathered}
$$

Then, by the variation of parameters formula, we have

$$
\left(w_{r}(t), w_{r t}(t)\right)=T(t, 0)\left(w_{r}(0), w_{r t}(0)\right)+\int_{0}^{t} T(t, \tau) G_{r}(\tau) d \tau
$$


where

$$
G_{r}(t):=\left(0, \eta_{r}\left(f\left(\|\nabla v(t)\|_{L^{2}\left(\mathbb{R}^{n}\right)}\right)-f\left(\|\nabla u(t)\|_{L^{2}\left(\mathbb{R}^{n}\right)}\right)\right) \Delta v(t)+f_{1}(t)\right) .
$$

Hence, applying Lemma 4.1 to (4.9) and taking into account (4.7), we obtain

$$
\begin{gathered}
\left\|\left(w_{r}(t), w_{r t}(t)\right)\right\|_{H^{2}\left(\mathbb{R}^{n}\right) \times L^{2}\left(\mathbb{R}^{n}\right)} \leq M e^{-\omega t}\left\|\theta_{2}-\theta_{1}\right\|_{H^{2}\left(\mathbb{R}^{n}\right) \times L^{2}\left(\mathbb{R}^{n}\right)} \\
+\widetilde{c}_{3} e^{-\omega t} \int_{0}^{t} e^{\omega \tau}\|\Delta v(\tau)\|_{L^{2}\left(\mathbb{R}^{n} \backslash B(0, r)\right)}\|w(\tau)\|_{H^{1}\left(\mathbb{R}^{n}\right)} d \tau \\
+\frac{\widetilde{c}_{3}}{r} e^{-\omega t} \int_{0}^{t} e^{\omega \tau}\|w(\tau)\|_{H^{4}\left(\mathbb{R}^{n}\right)} d \tau \\
\leq \widetilde{c}_{4}\left(e^{-\omega t}+\Pi_{r} e^{\left(\widetilde{c}_{1}-\omega\right) t}\right)\left\|\theta_{2}-\theta_{1}\right\|_{H^{2}\left(\mathbb{R}^{n}\right) \times L^{2}\left(\mathbb{R}^{n}\right)}, \quad \forall t \geq 0 \text { and } \forall r \geq 1,
\end{gathered}
$$

where

$$
\Pi_{r}:=\sup _{t \geq 0}\|\Delta v(t)\|_{L^{2}\left(\mathbb{R}^{n} \backslash B(0, r)\right)}+\frac{1}{r} .
$$

Then, the last inequality, together with (4.7), gives

$$
\begin{gathered}
\int_{0}^{t} e^{-\omega(t-\tau)}\left\|S(\tau) \theta_{2}-S(\tau) \theta_{1}\right\|_{H^{2}\left(\mathbb{R}^{n} \backslash B(0, r)\right) \times L^{2}\left(\mathbb{R}^{n} \backslash B(0, r)\right)} d \tau \\
\leq \widetilde{c}_{5}\left(e^{-\omega t}+\Pi_{r} e^{\left(\widetilde{c}_{2}-\omega\right) t}\right) \times \\
t\left\|\theta_{2}-\theta_{1}\right\|_{H^{2}\left(\mathbb{R}^{n}\right) \times L^{2}\left(\mathbb{R}^{n}\right)}, \forall t \geq 0 \text { and } \forall r \geq 1 .
\end{gathered}
$$

So, by (4.8) and (4.10), we have

$$
\begin{gathered}
\left\|S(t) \theta_{2}-S(t) \theta_{1}\right\|_{H^{4}\left(\mathbb{R}^{n}\right) \times H^{2}\left(\mathbb{R}^{n}\right)} \\
\leq \widetilde{c}_{6}\left(e^{-\omega t}+e^{-\omega t} t+\Pi_{r} e^{\left(\widetilde{c_{2}}-\omega\right) t} t\right)\left\|\theta_{2}-\theta_{1}\right\|_{H^{4}\left(\mathbb{R}^{n}\right) \times H^{2}\left(\mathbb{R}^{n}\right)} \\
+\widetilde{c}_{6} \sup _{0 \leq \tau \leq t}\left\|S(\tau) \theta_{2}-S(\tau) \theta_{1}\right\|_{H^{2}(B(0, r)) \times L^{2}(B(0, r))}, \forall t \geq 0, \forall r \geq 1 .
\end{gathered}
$$

From the compactness of $\mathcal{A}$, it follows that $\Pi_{r} \rightarrow 0$, uniformly with respect to the trajectories from $\mathcal{A}$, as $r \rightarrow \infty$. Thus, applying [20, Theorem 7.9.6], by (4.7) and (4.11), we obtain the finite dimensionality of $\mathcal{A}$.

\section{References}

[1] T. Cazenave, A. Haraux, An introduction to semilinear evolution equations, Oxford University Press, New York, 1998.

[2] F. Bucci, I. Chueshov, Long-time dynamics of a coupled system of nonlinear wave and thermoelastic plate equations, Discrete Contin. Dyn. Syst. 22 (2008) 557-586.

[3] L. Yang, Uniform attractor for non-autonomous plate equation with a localized damping and a critical nonlinearity, J. Math. Anal. Appl., 338 (2008) 1243-1254.

[4] S. Kolbasin, Attractors for Kirchoff's equation with a nonlinear damping coefficient, Nonlinear Analysis, 71 (2009) 2361-2371.

[5] A.Kh. Khanmamedov, Finite dimensionality of the global attractors for von Karman equations with nonlinear interior dissipation, Nonlinear Analysis, 66 (2007) 204-213.

[6] T.F. Ma, V. Narciso, Global attractor for a model of extensible beam with nonlinear damping and source terms, Nonlinear Analysis, 73 (2010) 3402-3412. 
[7] M. Potomkin, On transmission problem for Berger plates on an elastic base, Journal of Mathematical Physics, Analysis, Geometry, 7 (2011) 96-102.

[8] M. Potomkin, A nonlinear transmission problem for acompound plate with thermoelastic part, Math. Methods Appl. Sci., 35 (2012) 530-546.

[9] I. Chueshov, S. Kolbasin, Long-time dynamics in plate models with strong nonlinear damping, Commun. Pure Appl. Anal., 11 (2012) 659-674.

[10] T.F. Ma, V. Narciso, M.L. Pelicer, Long-time behavior of a model of extensible beams with nonlinear boundary dissipations, J. Math. Anal. Appl., 396 (2012) 694-703.

[11] M. A. Jorge Silva, V. Narciso, Long-time behavior for a plate equation with nonlocal weak damping, Differential and Integral Equations, 27 (2014) 931-948.

[12] M. Efendiev, S. Zelik, The attractor for a nonlinear reaction-diffusion system in an unbounded domain, Comm. Pure Appl. Math., 54 (2001) 625-688.

[13] A.Kh. Khanmamedov, Existence of a global attractor for the plate equation with a critical exponent in an unbounded domain, Applied Mathematics Letters, 18 (2005) 827-832.

[14] A.Kh. Khanmamedov, Global attractors for the plate equation with a localized damping and a critical exponent in an unbounded domain, J.Differential Equations, 225 (2006) 528-548.

[15] G. Yue, C. Zhong, Global attractors for plate equations with critical exponent in locally uniform spaces, Nonlinear Analysis, 71 (2009) 4105-4114.

[16] A.Kh. Khanmamedov, A global attractors for plate equation with displacement-dependent damping, Nonlinear Analysis, 74 (2011) 1607-1615.

[17] H.M. Berger, A new approach to the analysis of large deflections of plates, J. Appl. Mech., 22 (1955) 465-472.

[18] J. Ball, Global attractors for semilinear wave equations, Discr. Cont. Dyn. Sys., 10 (2004) $31-52$.

[19] A.Kh. Khanmamedov, Global attractors for von Karman equations with nonlinear interior dissipation, J. Math. Anal. Appl., 318 (2006) 92-101.

[20] I. Chueshov, I. Lasiecka, Von Karman Evolution Equations: Well-posedness and long-time dynamics, Springer, Berlin, 2010.

[21] P.G. Geredeli, J.T. Webster, Erratum to: Decay rates to equilibrium for nonlinear plate equations with degenerated geometrically-constrained damping, Appl. Math. Opt., 70 (2014) 565-566.

[22] H.L. Royden, Real Analysis, Macmillan, New York, 1968.

[23] A.Kh. Khanmamedov, Global attractors for 2-D wave equations with displacement dependent damping, Math. Methods Appl. Sci., 33 (2010) 177-187.

[24] A.V. Babin, M.I. Vishik, Attractors for evolution equations, North-Holland, Amsterdam, 1992.

Department of Mathematics,Faculty of Science, Hacettepe University, Beytepe 06800, Ankara, Turkey

E-mail address: zarat@hacettepe.edu.tr

Department of Mathematics,Faculty of Science, Hacettepe University, Beytepe 06800, Ankara, Turkey

E-mail address: azer@hacettepe.edu.tr

Department of Mathematics,Faculty of Science, Hacettepe University, Beytepe 06800, Ankara, Turkey

E-mail address: semasimsek@hacettepe.edu.tr 